\title{
Review of: "SARS-CoV-2 exposure in wild white-tailed deer (Odocoileus virginianus)"
}

\author{
Joaquin Vicente ${ }^{1}$ \\ 1 Universidad de Castilla La Mancha
}

Potential competing interests: The author(s) declared that no potential competing interests exist.

Limited work has been done on coronaviruses in white-tailed deer. Overall, the results indicate that certain white-tailed deer populations in several States were exposed to SARS-CoV-2. The commercially available SARS-CoV-2 antibody screening test used here is known to be highly specific (accurate) for use in other species. Although this has not been validated on deer, authors also tested a subset of samples using a different test specific to SARS-CoV-2. Both analyses resulted in identical findings. In addition, authors analyzed samples collected before and after SARS-CoV-2 was detected in humans in the Country. Minor:

- Paragraph 3: mention artificial feeding a overabundance in certain areas, including increased intraspecific contact in such points, and potential indirect contact with human (highest prevalence detected in ichigan, where these paractices are common, e.g. implication for bTB)

- Provide citation for the second test 